\title{
Physicochemical Interactions of Metformin Hydrochloride and Glibenclamide with Several Health Foods
}

\author{
Kazunari IWAO, ${ }^{a, b}$ Rushiana Tokie KAWAI, ${ }^{a}$ Masako ODA, ${ }^{a}$ \\ Michiya KOBAYASHI, ${ }^{a}$ and Hiroshi SAITOH ${ }^{*, a}$ \\ ${ }^{a}$ Department of Pharmaceutics, Faculty of Pharmaceutical Sciences, Health Sciences University \\ of Hokkaido, 1757 Kanazawa, Ishikari-Tobetsu, 061-0293, Japan, and bepartment \\ of Pharmacy, Health Sciences University of Hokkaido Hospital, \\ 2-5-1 Ainosato, Kita-ku, Sapporo 002-8072, Japan
}

(Received January 10, 2008; Accepted May 1, 2008)

\begin{abstract}
In this study, as an approach to possible pharmacokinetic interactions between antidiabetic agents and health foods, we investigated the binding of metformin and glibenclamide to aojiru, kurosu, and blueberry extract using a dialysis method. The permeation of metformin from the inner to the outer side of the dialysis tube during 90 min was not altered in the presence of aojiru or kurosu, and slightly decreased by $20 \%$ compared with the control in the presence of blueberry extract, indicating that the interactions between metformin and these three health foods are limited. On the other hand, when these three health foods were added to inner medium together with glibenclamide, they significantly lowered the outer glibenclamide concentrations. The magnitude of the decrease in the outer glibenclamide concentrations was the most extensive in the presence of kurosu (60\%), followed by aojiru (36\%) and blueberry extract (45\%). The present results suggest that metformin and glibenclamide interact differently with aojiru, kurosu, and blueberry extract and that these three health foods might modulate the absorption of glibenclamide in vivo.
\end{abstract}

Key words — metformin hydrochloride; glibenclamide; physicochemical interaction; aojiru; kurosu; blueberry extract

\section{INTRODUCTION}

Reflecting the recent health boom, there is increasing use of a variety of health foods by a wide range of age-groups in Japan. Some health foods are known to exert pharmacologic activities against various diseases such as hypertension, hyperlipidemia, and diabetes mellitus (DM), although their potential benefits are far less marked than those of therapeutic agents. For example, dietary fiber is known to lower blood sugar levels, ${ }^{1,2)}$ and the Japan Diabetes Society has recommended the intake of dietary fiber as a grade B treatment for DM. Moreover, the effectiveness in lowering blood sugar levels of five functional health foods (indigestible dextrin, L-arabinose, guava polyphenol, wheat albumin, and tohchi extract) has been certified by the Japanese government. ${ }^{3)}$ It is likely that these circumstances will encourage diabetic patients and those on the border of DM to use functional health foods.

On the other hand, it is possible that the intake of large amounts of a particular health food could lead to an excessive supply of sugar and calories to diabet-

\footnotetext{
*e-mail: saitoh@hoku-iryo-u.ac.jp
}

ic patients ${ }^{4)}$ and that hypoglycemia could be induced unexpectedly when a patient consumes a health food to which an antidiabetic agent was added.5,6) Moreover, attention should be paid to possible pharmacokinetic interactions between antidiabetic agents and health foods. It was reported previously that simultaneous administration of glibenclamide and glucomannan to healthy Japanese volunteers caused significantly lowered glibenclamide concentrations in the blood, indicating possible interference in the intestinal absorption of the antidiabetic drug by glucomannan. ${ }^{7)}$ Further, it has been reported that guar gum modulated the absorption rate of metfor$\min { }^{8)}$

According to several recent studies, approximately half of diabetic patients consume certain health foods for the purpose of lowering blood sugar levels or controlling their physical condition..$^{9,10)}$ Similarly, our recent survey of diabetic outpatients at the Health Sciences University of Hokkaido Hospital showed that about $54 \%$ of diabetic outpatients had consumed health foods (unpublished data), with the most popular health foods being aojiru, kurosu, and blueberry extract. Our survey made clear that a small number of outpatients consume such health foods 
simultaneously with prescribed antidiabetic agents. Metformin hydrochloride and sulfonylureas (glibenclamide, glimepiride, and gliclazide) were frequently administered to outpatients at the Health Sciences University of Hokkaido Hospital. However, little information is available on the interactions between these antidiabetic agents and health foods such as aojiru, kurosu, and blueberry extract.

In this study, as an approach to the pharmacokinetic interactions between antidiabetic agents and health foods, we undertook a preliminary investigation of the binding of metformin and glibenclamide to aojiru, kurosu, and blueberry extract using a dialysis method.

\section{MATERIALS AND METHODS}

Materials Metformin hydrochloride and glibenclamide were purchased from MP Biochemical (Solon, OH, USA) and Wako Pure Chemical Industries (Osaka, Japan), respectively. Commercially available aojiru, blueberry extract, and kurosu were purchased from Fancl Co. (Yokohama, Japan), Japan Sana Co. (Sakura, Chiba, Japan), and Orihiro Co. (Takasaki, Gunma, Japan), respectively. Other reagents were of the highest grade available.

Binding Experiments Using Dialysis Methods

One pack $(3.5 \mathrm{~g})$ of freeze-dried aojiru was suspended in $100 \mathrm{ml}$ of Tyrode's solution $(\mathrm{NaCl} 137$ $\mathrm{mM}, \mathrm{KCl} 3 \mathrm{mM}, \mathrm{CaCl}_{2} 2 \mathrm{mM}, \mathrm{MgCl}_{2} 1 \mathrm{mM}, \mathrm{NaHCO}_{3}$ $12 \mathrm{mM}, \mathrm{NaH}_{2} \mathrm{PO}_{4} 0.4 \mathrm{mM}$, D-glucose $6 \mathrm{mM}, \mathrm{pH} 6.5$ ). Blueberry extract was diluted in advance with an equal volume of Tyrode's solution, whereas no predilution was performed for kurosu. Drug solutions were prepared with Tyrode's solution and mixed with an equal volume of aojiru suspension, blueberry extract, or kurosu just prior to dialysis. The $\mathrm{pH}$ of the three test solutions including aojiru, blueberry extract, or kurosu was approximately $6.5,3.5$, and 3.0, respectively. As a control, drug solutions were mixed with an equal volume of Tyrode's solution. The final concentration of drugs was either 50 or $100 \mu \mathrm{M}$.

The dialysis tube (Spectra/Por membrane, MWCO 1000 , diameter $1.5 \mathrm{~cm}$ ) purchased from Spectrum Medical Industries Inc. (Houston, TX, USA) was cut into lengths of about $7 \mathrm{~cm}$, soaked in Tyrode's solution, and then incubated for $10 \mathrm{~min}$ at $38^{\circ} \mathrm{C}$. Three milliliters of test solution with or without the health foods was introduced into the dialysis tubes. The tubes were then clamped and gently stirred in $150 \mathrm{ml}$ of Tyrode's solution. After 90 min, the outer medium $(5 \mathrm{ml})$ and inner solution $(2 \mathrm{ml})$ were collected for determination of drug concentration and kept frozen at $-30^{\circ} \mathrm{C}$ until assay.

HPLC Analysis The HPLC system consisted of a Shimadzu LC-10ATvp solvent delivery system (Shimadzu, Kyoto, Japan) equipped with a Shimadzu SPD-10Avp detector. A Cosmosil 5C18ARII column $(4.6 \times 150 \mathrm{~mm}$, Nacalai Tesque, Kyoto, Japan) was used as the stationary phase. The mobile phases were $\mathrm{NaH}_{2} \mathrm{PO}_{4} 40 \mathrm{~mm}$ with 1-octanesulfonic acid sodium salt $5 \mathrm{mM}(\mathrm{pH} 6) /$ acetonitrile $(9: 1)$ for metformin and $\mathrm{KH}_{2} \mathrm{PO}_{4} 50 \mathrm{~mm} /$ acetonitrile $(1: 1)$ for glibenclamide. The flow rate was set at $1.0 \mathrm{ml} /$ min for both drugs. The wavelength for detection of metformin and glibenclamide was $234 \mathrm{~nm}$ and 230 $\mathrm{nm}$, respectively. Column temperature was set at $50^{\circ} \mathrm{C}$.

Statistical Analysis Data are given as mean with S.D. of three experiments. All data were analyzed with one-way analysis of variance (ANOVA); the group means were compared using Tukey's test for multiple comparisons. The level of significance was set at $p<0.05$.

\section{RESULTS}

Binding of Metformin to Aojiru, Blueberry Extract, and Kurosu The diffusion into the outer medium of metformin with or without the three health foods tested is shown in Fig. 1. The equilibrium concentration was expected to be approximately 2 $\mu \mathrm{M}$ in the case of metformin. In the absence of any health food, the metformin concentration in the outer medium was approximately $1.5 \mu \mathrm{M}$ after $90 \mathrm{~min}$, reaching $c a .70 \%$ of the equilibrium concentration. When metformin was introduced into the dialysis tubes with aojiru and kurosu, its concentration in the outer medium after 90 min was almost the same as that of the control, implying that metformin did not interact with aojiru or kurosu. In the presence of blueberry extract, however, the metformin concentration in the outer medium significantly decreased to approximately $1.2 \mu \mathrm{M}$ (80\% of control), indicating that there might be an interaction between metformin and blueberry extract.

Binding of Glibenclamide to Aojiru, Blueberry Extract, and Kurosu Figure 2 shows the diffusion of glibenclamide with or without the three health foods into the outer medium. The equilibrium concentra- 
Metformin concentration $(\mu \mathrm{M})$

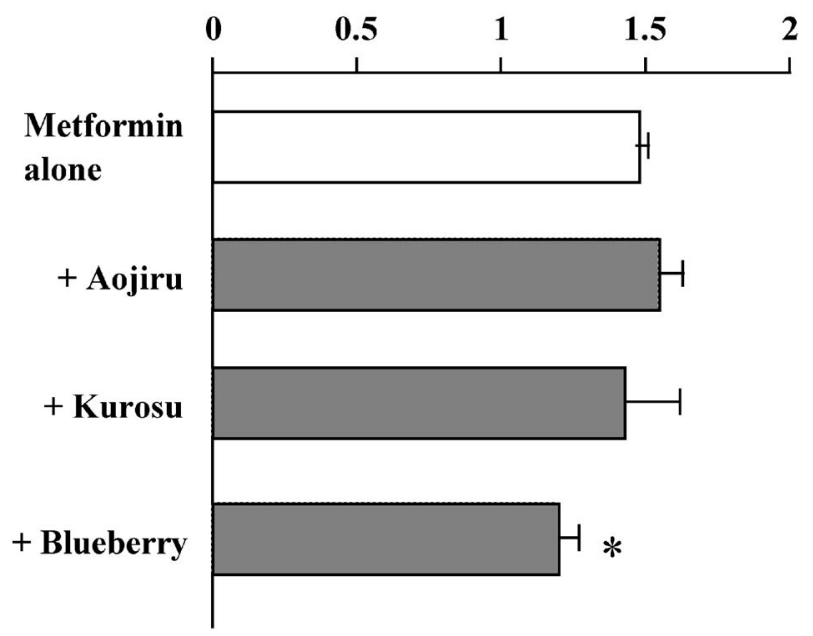

Fig. 1. Effects of Aojiru, Kurosu, and Blueberry Extract on the Diffusion of Metformin into the Outer Dialysis Medium in $90 \mathrm{~min}$

The mixture $(3 \mathrm{ml})$ of metformin hydrochloride and aojiru, kurosu, or blueberry extract was introduced into a dialysis tube and then gently stirred in $150 \mathrm{ml}$ of Tyrode's solution ( $\mathrm{pH} 6.5$ ). After $90 \mathrm{~min}$, an aliquot of the outer medium was collected for determination of the metformin concentration. The initial concentration of metformin in the inner dialysis medium was 100 $\mu \mathrm{M}$. Each column represents the mean with S.D. of three experiments. ${ }^{*} p<$ 0.05 , significantly different from metformin alone.

Glibenclamide concentration $(\mu \mathrm{M})$

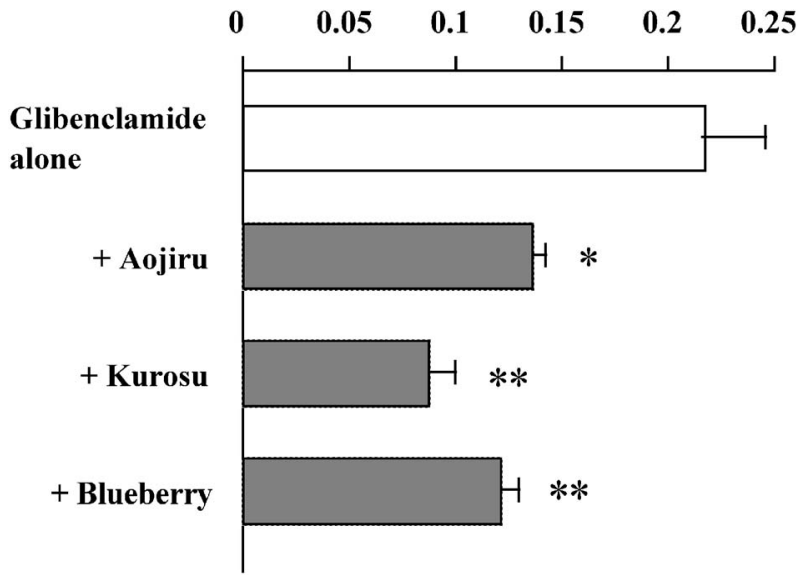

Fig. 2. Effects of Aojiru, Kurosu, and Blueberry Extract on the Diffusion of Glibenclamide into the Outer Dialysis Medium in $90 \mathrm{~min}$

The mixture $(3 \mathrm{ml})$ of glibenclamide and aojiru, kurosu, or blueberry extract was introduced into a dialysis tube and then gently stirred in $150 \mathrm{ml}$ of Tyrode's solution ( $\mathrm{pH}$ 6.5). After $90 \mathrm{~min}$, an aliquot of outer medium was collected for determination of the glibenclamide concentration. The initial concentration of glibenclamide in the inner dialysis medium was $50 \mu \mathrm{M}$. Each column represents the mean with S.D. of three experiments. ${ }^{*} p<0.05$, ${ }^{* *} p<0.01$, significantly different from glibenclamide alone.

tion of glibenclamide was expected to be approximately $1 \mu \mathrm{M}$. In the absence of any health food, the glibenclamide concentration in the outer medium was approximately $0.22 \mu \mathrm{M}$ after $90 \mathrm{~min}$ and remained at $22 \%$ of the equilibrium concentration. The value was much smaller than that for metformin. In the present study, dialysis tubes with a molecular cutoff of 1000 were used. Accordingly, it was considered that the permeation of glibenclamide with a molecular weight of 494.0 was more difficult than that of metformin with its smaller molecular weight. When glibenclamide was introduced into the dialysis tubes with aojiru, kurosu, and blueberry extract, its concentration in the outer medium was approximately $0.14 \mu \mathrm{M}$, $0.09 \mu \mathrm{M}$, and $0.12 \mu \mathrm{M}$, respectively, after $90 \mathrm{~min}$, all of which are significantly less than the control value, suggesting that glibenclamide is capable of interacting with these three health foods. The magnitude of the decrease in the glibenclamide concentrations in the outer medium was most marked in the presence of kurosu, followed by blueberry extract and aojiru.

\section{DISCUSSION}

Previous studies on the pharmacokinetic interactions of antidiabetic agents with several health foods generally reported decreased or delayed absorption of these agents. ${ }^{\left.7{ }^{78}\right)}$ A likely mechanism is the binding (or adsorption) of the antidiabetic agents to the ingredients of the health foods. Extensive modulation of intestinal absorption via such physicochemical interactions might lead to problems in DM treatment.

As aojiru is a health food made from green vegetables such as kale, ashitaba, mulberry leaf, barley grass, etc. and commercial aojiru products often contain large amounts of vitamin $\mathrm{K}$ and metallic cations. Thus aojiru might interact with warfarin and fluoroquinolones. ${ }^{11,12)}$ Aojiru is also a fiber-rich product and includes an insoluble component; therefore the binding (or adsorption) of some drugs, including antidiabetic agents, to aojiru seems likely. However, there was no interaction between metformin and aojiru in this study (Fig. 1). In the case of glibenclamide, on the other hand, its diffusion into the outer medium after 90 min was significantly less in the presence of than in the absence of aojiru, indicating the binding (or adsorption) of glibenclamide to some insoluble component of aojiru. This result implies that aojiru might modulate the absorption of glibenclamide when they are consumed together.

There have been few reports describing the interactions of kurosu and blueberry extract with antidiabetic agents. However, as both health foods contain vari- 
ous ingredients, ${ }^{13,14)}$ it is possible that some ingredients interact with antidiabetic agents and modulate their absorption. As shown in Fig. 1, no significant change was observed in the metformin concentrations in the outer medium when the agent was added to the dialysis tube in the presence and absence of kurosu, indicating that metformin does not interact with the ingredients in kurosu. On the other hand, there was a small but significant decrease in the metformin concentration in the outer medium when the antidiabetic agent was introduced into the dialysis tube together with the blueberry extract. However, the overall data obtained in this study suggest that the consumption of aojiru, kurosu, or blueberry extracts by diabetic patients receiving metformin treatment would not result in any clinically relevant pharmacokinetic interactions.

In contrast, glibenclamide underwent potent interactions with kurosu and blueberry extract (Fig. 2). Kurosu is a dark brown liquid whereas blueberry extract is a dark blue liquid. The centrifugation of kurosu and blueberry extract at $10000 \times \mathrm{g}$ for $30 \mathrm{~min}$ did not give any precipitate. Therefore it seems that the interaction between glibenclamide and kurosu and blueberry extract did not involve binding (or adsorption) to insoluble ingredients but rather complexation or chelating with water-soluble ones. If metformin and glibenclamide interact with the same ingredient in blueberry extract, the decrease in the outer glibenclamide concentration was expected to be more marked in the case of blueberry extract. Interestingly, however, the present results suggest that the interaction between glibenclamide and kurosu was more potential than those with aojiru and blueberry extract. The magnitude of the decrease in the outer glibenclamide concentration was approximately $60 \%$ in the presence of kurosu. To the best of our knowledge, no pharmacokinetic interaction between glibenclamide and kurosu has been reported in the literature. Therefore it is very important to determine the extent to which kurosu is capable of modulating the intestinal absorption of glibenclamide. It is also necessary to assess whether kurosu interacts with other clinically relevant drugs. Recently, Watanabe et $a l .{ }^{15)}$ reported that the interaction between drugs and dietary fiber was $\mathrm{pH}$ sensitive. Moreover, as indicated previously, as guar gum did not influence the absorption of glipizide, ${ }^{16)}$ a sulfonylurea, the interaction between antidiabetic agents and health foods might be drug specific. The physicochemical interactions between health foods and drugs would also depend on the concentrations tested. Therefore further in vitro and in vivo studies are essential to clarify the clinical relevance of the physicochemical interactions between glibenclamide and the three health foods tested.

In conclusion, our preliminary study suggests that the two antidiabetic agents metformin and glibenclamide interacted differently with aojiru, kurosu, and blueberry extract. The absorption of glibenclamide, but not of metformin, is likely to be modulated by these three health foods through physicochemical interactions.

\section{REFERENCES}

1) Chandalia M., Garg A., Lutjohann D., von Bergmann K., Grundy S. M., Brinkley L. J., N. Engl. J. Med., 342, 1392-1398 (2000) .

2) Tabatabai A., Ki S., Clin. Excell. Nurse Pract., 4, 272-276 (2000).

3) Itoh H., Biomed. Ther., 39, 976-980 (2005).

4) Yamashita S., Odawara M., Practice, 20, 404410 (2003).

5) Kumasaka K., Kojima T., Doi K., Satoh S., Yakugaku Zasshi, 123, 1049-1054 (2003).

6) Kamimura H., Yoshida M., Tominaga H., Miyazaki S., Fujita T., Sasaki H., Karube Y., Jpn. J. Pharm. Health Care Sci., 32, 407-413 (2006).

7) Shima K., Tanaka A., Ikegami H., Tabata M., Sawazaki N., Kumahara Y., Horm. Metab. Res., 15, 1-3 (1983).

8) Gin H., Orgerie M. B., Aubertin J., Horm. Metab. Res., 21, 81-83 (1989).

9) Kudoh M., Kanda M., Asakura S., Matsui F., Nogami Y., Makino J., Umeno Y., Practice, 22, 596-599 (2005).

10) Homma H., Hashimoto Y., Miyazaki N., Matsumoto R., Sasaki M., Jpn. J. Pharm. Health Care Sci., 33, 457-462 (2007).

11) Sakamaki N., Nakazato M., Matsumoto H., Hagino K., Yasuda K., Nagayama T., Shokuhin Eiseigaku Zasshi, 47, 85-88 (2006).

12) Tsunoda M., Egawa T., Sejima H., Sejima M., Tominaga K., Kataoka Y., Shuto H., Jpn. J. Health Care Sci., 33, 534-539 (2007).

13) Shimoji Y., Tamura Y., Nakamura Y., Nanda K., Nishidai S., Nishikawa Y., Ishihara N., Uenakai K., Ohigashi H., J. Agric. Food 
Chem., 50, 6501-6503 (2002).

14) Dugo P., Mondello L., Errante G., Zappia G., Dugo G., J. Agric. Food Chem., 49, 39873992 (2001).

15) Watanabe S., Inoue N., Imai K., Suemaru K.,
Araki H., Aimoto T., Jpn. J. Pharm. Health Care Sci., 32, 221-226 (2006).

16) Huupponen R., Karhuvaara S., Seppala P., Eur. J. Clin. Pharmacol., 18, 717-719 (1985). 